Artigo

\title{
Eventos Extremos Diários de Chuva no Nordeste do Brasil e Características Atmosféricas
}

\author{
José Maria Brabo Alves ${ }^{1}$, Emerson Mariano da Silva ${ }^{1}$, Sérgio Souza Sombra ${ }^{1}$, Augusto César \\ Barros Barbosa ${ }^{1}$, Antônio Carlos Santana dos Santos ${ }^{1}$, Marcos Antonio Tavares Lira ${ }^{2}$ \\ ${ }^{1}$ Departamento de Física, Centro de Tecnologia, Universidade Estadual do Ceará, \\ Fortaleza, CE, Brasil. \\ ${ }^{2}$ Departamento de Engenharia Elétrica, Universidade Federal do Piauí, Teresina, PI, Brasil
}

Recebido em 3 de Fevereiro de 2016 - Aceito em 3 de Março de 2017

\begin{abstract}
Resumo
Este estudo investigou as principais características atmosféricas associadas a eventos extremos de precipitação com uma defasagem de três dias antes da ocorrência desses extremos. Os extremos foram definidos quando na maior parte dos pontos de grade sobre o Nordeste do Brasil ( $2^{\circ} \mathrm{S}-12^{\circ} \mathrm{S}$ e $\left.45^{\circ} \mathrm{W}-37^{\circ} \mathrm{W}\right)$, as chuvas diárias foram iguais ou superiores a $50 \mathrm{~mm}$. Foram utilizados dados diários de precipitação de um conjunto de estações pluviométricas distribuídas sobre o NEB, provenientes das redes observacionais pertencentes ao Instituto Nacional de Meteorologia, Agência Nacional de Águas e Núcleos Estaduais de Meteorologia e Recursos Hídricos, cobrindo o período de 1974-2003. Estes dados foram interpolados espacialmente, usando a técnica do inverso do quadrado da distância, para uma grade regular com resolução de $1^{\circ}$ de latitude e longitude (aproximadamente $111 \mathrm{~km}$ ). Para análises das características atmosféricas foram usados dados de reanálises de variáveis atmosféricas como: vento zonal meridional, temperatura do ar, umidade relativa e outras das reanálises do NCEP-NOAA desde a superfície até $200 \mathrm{mb}$. Os resultados mais expressivos mostraram que três dias antes dos eventos de precipitação considerados extremos, há uma incursão de ar frio para a área central do NEB, intensificando um dia antes dos dias dos eventos, com anomalias inferiores a $-4{ }^{\circ} \mathrm{C}$, que não aparecem para os eventos considerados de pouca precipitação sobre a região.
\end{abstract}

Palavras-chave: composição de eventos, chuva intensa.

\section{Extreme Rainfall Events in Northeast Brazil and Atmospheric Characteristics}

\begin{abstract}
This study investigated the means characteristics atmospherics associated with extreme precipitation events with until three-day delay before the occurrence of such extremes. The extreme events were chose when in most grid points on the Northeast of Brazil $\left(2^{\circ} \mathrm{S}-12^{\circ} \mathrm{S}\right.$ and $\left.45^{\circ} \mathrm{W}-37^{\circ} \mathrm{W}\right)$, daily rainfall was equal or more than $50 \mathrm{~mm}$. Daily data precipitation were of a set of rainfall stations distributed over NEB, from the observational networks of the National Institute of Meteorology, National Water Agency and Meteorology and Water Resources Regional's Centers, covering the period 1974-2003. These data were interpolated spatially using the technique of Inverse Square of the distance to a regular grid with a resolution of $1^{\circ}$ in latitude and longitude (about $111 \mathrm{~km}$ ). For analyzes of atmospheric features were used in atmospheric variables reanalysis data as southern zonal wind, air temperature, relative humidity and other reanalysis of NCEP-NOAA from the surface until $200 \mathrm{mb}$. The most significant results showed that three days before of the precipitation events considered extreme, there is a cold air incursion into the central area of the NEB, intensifying a day before of the events days, with lower anomalies below $-4{ }^{\circ} \mathrm{C}$, which do not appear to events considered of low rainfall over the region.
\end{abstract}

Keywords: composition of events, intense rainfall.

Autor de correspondência: José Maria Brabo Alves, braboalves@gmail.com. 


\section{Introdução}

A Região Nordeste do Brasil (NEB) tem uma alta variabilidade interanual e intrasazonal de precipitação, com a influência térmica dos oceanos Pacífico e Attântico Tropical controlando anos de seca e enchentes na região (Ropelewisk e Jones, 1987; Ropelewisk e Halpert, 1997, Moura e Shukla, 1996).

Uma característica interessante dessa variabilidade interanual da precipitação no NEB, comparado a áreas vizinhas com a Região Amazônica foi mostrado por Kousky (1979). Diferenças marcantes são observadas nessas regiões, em $10^{\circ} \mathrm{S}-40^{\circ} \mathrm{W}$ e $0^{\circ}-65^{\circ} \mathrm{W}$ com totais anuais e desvios padrões de $530 \mathrm{~mm}$ e $97 \mathrm{~mm}$ e $2450 \mathrm{~mm}$ e $80 \mathrm{~mm}$, respectivamente (Liebmann et al., 2011).

Muitos estudos sobre a variabilidade interanual e intrassazonal da precipitação no NEB têm sido executados nestas últimas décadas (Hasterath e Greischar, 1993b; Moura, 1981; Nobre e Shukla, 1996). O período chuvoso do NEB, na sua porção mais semi-árida, é curto concentrando-se nos primeiros seis meses do ano, com um período mais seco nos últimos seis meses, refletindo no acumulo de água a superfície, que escoa para os reservatórios hídricos da região (Alves et al., 2009).

Esta variabilidade pluviométrica no NEB, com ênfase a sua região semi-árida, está diretamente acoplada à evolução térmica anual no Atlântico Tropical, cujo modo chamado de dipolo de TSM, associa-se ao acoplamento e migração norte-sul da Zona de Convergência Intertropical (ZCIT) e circulação atmosférica de baixos níveis (variações nos ventos alísios de nordeste e sudeste), que definem suas condições climáticas extremas. A importância deste modo também chamado de gradiente meridional define anos de secas ou enchentes no NEB. A ocorrência concomitante de anomalias de TSM positivas (negativas) de TSM nos setores norte (sul) do equador no Atlântico Tropical, e posicionamento da ZCIT mais ao norte (sul) da sua climatologia, alísios de nordeste (sudeste) mais fracos (fortes) que seus valores médios, em geral, associam-se com anos de precipitação escassa no NEB. Em condições térmicas contrárias são observadas características adversas de precipitação (Nobre e Shukla, 1996, Hastenrath and Greischar, 1993b, Uvo et al., 1998).

Relacionado, a investigação dos principais fatores associados à variação da ocorrência diária de precipitação no NEB, isto é diferença de extremos de intensidade que condicionam esses totais anuais, esse assunto pouco tem sido um fator de pesquisa. Nos últimos anos, principalmente, as chuvas diárias intensas tem causado transtornos e perdas econômicas e sociais em várias do NEB (Alves et al., 2006).

Estes eventos extremos discretos de precipitação são modeladores dos totais de precipitação no NEB como mostrados por vários estudos (Ramos, 1975; Kousky, 1979; De Souza et al., 2005 e Rao et al., 2007). Liebmann et al., 2011, ilustra a natureza discreta da precipitação no NEB em dois pontos com coordenadas geográficas localizadas no norte e centro-sul do NEB. Durante a estação chuvosa de 1985, meses de fevereiro a março, no ponto de coordenada próximo ao litoral $\left(5^{\circ} \mathrm{S}-37,5^{\circ} \mathrm{W}\right) 40 \%$ dos dias foram considerados secos, enquanto no ponto de coordenada mais ao $\operatorname{sul}\left(10^{\circ} \mathrm{S}, 40^{\circ} \mathrm{W}\right)$, nos meses de fevereiro a março, $85 \%$ dos dias classificados como secos.

Esses resultados de Liebmann et al., 2011, foram consistentes com os de Ramos (1975) e Moran (2007). Em seus resultados Ramos (1975) encontrou que seis a oito eventos de precipitação diária contribuem significativamente para o total anual de chuva no NEB, enquanto Moran et al. (2007) mostrou que a maioria dos postos pluviométricos no NEB tiveram mais que 35 dias com chuva anualmente. A atuação de vários tipos de sistemas atmosféricos tem sido usada como explicação para a ocorrência de eventos discretos de precipitação no NEB. Ramos (1975) mostrou que distúrbios que se movem para oeste com a mesma velocidade dos ventos na baixa troposfera estavam relacionados a esses eventos, e mais lentamente do que se esses estivessem associados a distúrbios tropicais. Evidência de influências de ondas subtropicais foi observada por Kousky (1979), mostrando uma propagação tipo onda na direção norte quando ocorreram eventos de chuva no NEB. A propagação de frentes frias para o norte, suas influências e seus remanescentes foram observadas por Kousky (1979) e Kousky e Ferreira (1981) quando da atuação desses eventos discretos de precipitação no NEB. Vale mencionar que estudos que mostram a propagação de frentes frias latitudes médias do Hemisfério se propagando para o norte/nordeste e suas influências em padrões climáticos na Amazônia e várias áreas da América do Sul em todas as estações do ano têm sido amplamente documentadas (Kusky por Kousky, 1981; Garreaud, 2000; Vera e Vigliarolo, 2000). Liebmann et al., 2011 argumenta que o sinal mais forte dessa influência, nas latitudes mais equatoriais, é verificado no campo de pressão e ventos a superfície e fraco nos campos de mistura e temperatura (Garreaud e Wallace, 1998; Rickembach et al., 2002). Kousky (1979) inferiu que a característica e propagação das frentes frias que se deslocam sobre o NEB, e não os seus números estão mais relacionados à intensidade desses eventos discretos de precipitação na região. Frentes que se deslocam mais lentamente, possivelmente associadas com o desenvolvimento de ondas equatoriais, estão relacionados a totais mais expressivos de precipitação.

O estudo de Liebmann et al. (2011) é mais recente com o objetivo de explicar alguns dos principais mecanismos atmosféricos associados a dias de chuva forte no NEB, considerado com um dia chuvoso quando a chuva excedeu a 2 desvios padrões do total médio diário anual, nos seus dois pontos de estudo, ponto de coordenada próximo ao litoral $\left(5^{\circ} \mathrm{S}-37,5^{\circ} \mathrm{W}\right)$ e o ponto de coordenada mais ao sul $\left(10^{\circ} \mathrm{S}, 40^{\circ} \mathrm{W}\right)$. Em seus principais resultados, em $5^{\circ} \mathrm{S}-37,5^{\circ} \mathrm{W}$, as chuvas estiveram associados a distúr- 
bios que se propagaram de oeste, com características de ondas de Kelvin equatoriais (Liebmann et al., 2009). E em $10^{\circ} \mathrm{S}, 40^{\circ} \mathrm{W}$, esses eventos discretos de precipitação foram associados, principalmente, a influência de ondas de Rosby que propagam devido a incursão de frentes frias que se deslocam das proximidades do pólo sul na direção do equador.

O objetivo desse estudo foi identificar as principais características da atmosfera, em dias com extremos de precipitação (chuva forte: intensidade maior ou igual a $50 \mathrm{~mm}$ e chuva fraca com intensidade menor ou igual a $5 \mathrm{~mm}$ ), com algumas análises de variáveis termodinâmicas na atmosfera, somente no período de fevereiro a maio (quadra chuvosa do setor norte do NEB) de 1974-2003. Os resultados encontrados poderão ser de extrema utilidade na rotina operacional de centros de meteorologia de previsão de tempo da região semiárida do NEB.

\section{2 - Dados Utilizados}

Foram utilizados dados diários de precipitação de um conjunto de estações pluviométricas distribuídas sobre o NEB (Fig. 1), provenientes das redes observacionais pertencentes ao Instituto Nacional de Meteorologia, Agência Nacional de Águas e Núcleos Estaduais de Meteorologia e Recursos Hídricos, cobrindo o período de 1974-2003. Estes dados foram interpolados espacialmente, usando a técnica do inverso do quadrado da distância, para uma grade regular com resolução de $1^{\circ}$ em latitude e longitude (aproximadamente $111 \mathrm{~km}$ ). Detalhes sobre os procedimentos de

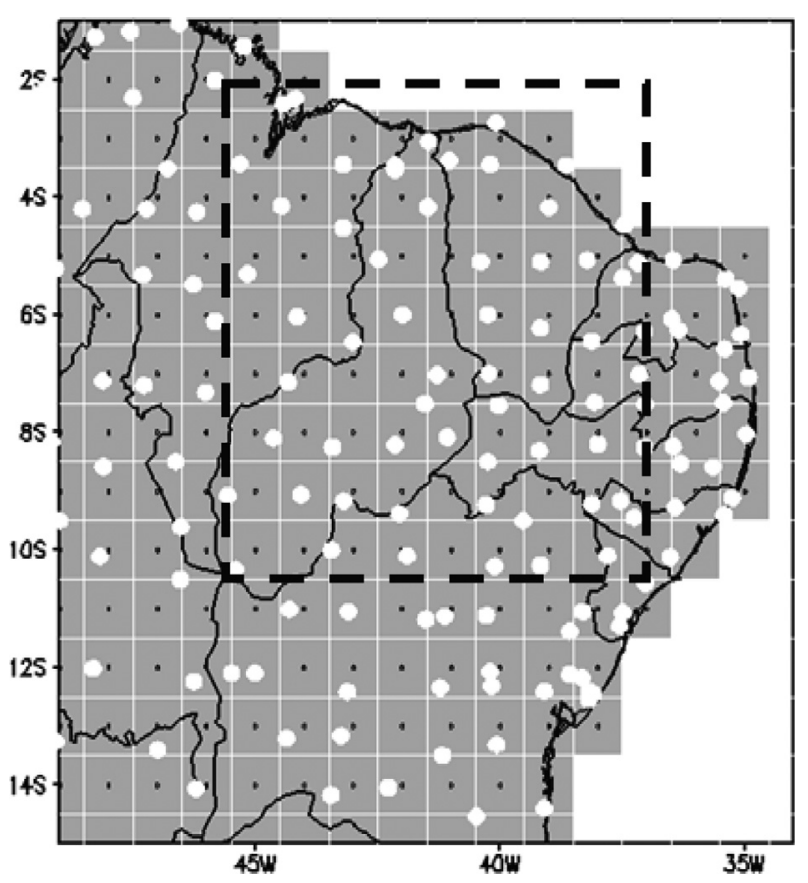

Figura 1 - Estações pluviométricas (pontos em branco) e grade regular de $1^{\circ} \times 1^{\circ}$ (pontos em preto) sobre a região do NEB. O retângulo destacado em linha pontilhada indica o setor norte do Nordeste (SNNEB) usada na investigação do sinal intrassazonal. controle de qualidade destes dados, bem como comparações com outras bases de dados encontram-se descritos em Souza et al. (2005).

As variáveis atmosféricas das reanálises do National Center for Environment Prediction (NECP) foram usadas diariamente entre 1 de fevereiro a 31 de maio no período de 1971-2003. As variáveis usadas foram: componente zonal e meridional do vento $\left(\mathrm{m} . \mathrm{s}^{-1}\right)$, temperatura $\left({ }^{\circ} \mathrm{C}\right)$, pressão (hpa), movimento vertical (hpa. $\left.\mathrm{s}^{-1}\right)$ e vorticidade $\left(\mathrm{s}^{-1}\right)$. Detalhes sobre esses dados de reanálises podem sem encontrados em Kalnay et al. (1996). Estas variáveis foram usadas nos níveis padrões de pressão de 1000, 925, 850, 700, 500, 400, 300 e 200 hpa. Para auxiliar nas análises foram calculadas variáveis atmosféricas derivadas como: divergência do vento $\left(\mathrm{m} \cdot \mathrm{s}^{-1}\right)$ e umidade $\left(\mathrm{m} \cdot \mathrm{g} \cdot \mathrm{kg}^{-1} \cdot \mathrm{s}^{-1} 1\right)$, advecção de temperatura $\left({ }^{\circ} \mathrm{C} \cdot \mathrm{s}^{-1}\right)$.

\section{Metodologia}

Para classificar os dias com chuva intensa e leve foi considerado aquele quando mais de $60 \%$ dos pixeis $\left(517.482 \mathrm{~km}^{2}\right)$ da área entre $45^{\circ} \mathrm{W}-37^{\circ} \mathrm{W}$ e $12^{\circ} \mathrm{S}-2^{\circ} \mathrm{S}$ (Fig. 1) tiveram um total de chuva diária igual ou superior a $50 \mathrm{~mm}$ e igual ou inferior a $5 \mathrm{~mm}$. Essa área tem dez pontos de grade na direção norte-sul (latitude) e sete na direção (oeste-leste) longitude com uma área aproximada de $862.470 \mathrm{~km}^{2}$.

Após essa classificação foi calculada uma média e a anomalia, do dia central classificado como de chuva intensa ou de chuva leve, de três dias defasados e após um dia do dia central, para todas as variáveis em cada ponto de grade da Fig. 1. Em seguida foi feita média entre 1000-850 hpa, entre 700-500 hpa e 300-200 hpa, considerados como de baixa, média e alta troposfera. Para a variável umidade específica sua advecção e fluxo de vapor estes cálculos foram feitos apenas na baixa troposfera, em função de que mais de $80 \%$ da umidade atmosférica se concentra nos baixos níveis da atmosfera. A climatologia usada no cálculo da anomalia dessas variáveis foi calculada usando o período de 1974-2003.

Foram plotados mapas espaciais das anomalias de circulação (ventos) e das variáveis citadas acima para cada 3 dias antes, no dia da precipitação mais intensa e menos intensa e um dia após, para que se possa identificar possíveis diferenças. Área desses mapas compreendeu uma região entre $90^{\circ} \mathrm{W}-10^{\circ} \mathrm{E}$ e $60^{\circ} \mathrm{S}-60^{\circ} \mathrm{N}$.

Para auxiliar nas análises foram plotados também diagramas de dispersão dos valores diários, média em uma área representativa do setor norte do NEB $-45^{\circ} \mathrm{W}-37^{\circ} \mathrm{W}$ e $12^{\circ} \mathrm{S}-2^{\circ} \mathrm{S}$, de variáveis como movimento vertical, divergência horizontal do vento e umidade, vorticidade na baixa e média atmosfera e o os valores de precipitação para os referidos dias de chuva intensa e leve para se possível identificar alguma relação magnitude da variável física e a intensidade da precipitação. Além disso, foram plotados esses diagramas relacionando também a área com diver- 
gência e movimento vertical negativa e advecção de temperatura positiva.

Neste estudo os dados diários não foram filtrados para eliminar influência de sistemas atmosféricos de baixa frequência, como por exemplo, Oscilação de Madden e Julian que tem influência na precipitação do NEB (Carvalho e Jones, 2004; De Souza e Ambrizzi, 2006). Os autores decidiram pela opção de não filtrar pelos resultados apresentados no artigo de Liebmann et al. (2011) que mostrou que a classificações de eventos (dias chuvosos) com sua metodologia usada foi similar quando este usou dados não filtrados e filtrados com um filtro de Lanczos passa baixa para remover períodos de frequência superior a 30 dias.

\section{Resultados e Discussão}

A Fig. 2 mostra as anomalias de ROL, ventos em $850 \mathrm{hPa}$ e geopotencial em $200 \mathrm{hPa}$ para dias $-3,-2,-1,0 \mathrm{e}$ +1 dos dias considerados chuvosos. Nota-se pelo campo de anomalias de ROL que se observam desde o dia -3, até o dia 0 , com uma diminuição nesse dia de anomalias negativas de ROL desde o Pacífico Leste, passando pela Amazônia. Essa característica é típica da propagação de ondas de Kelvin intrassazonais, como mostrado em Liebmann et al. (2009 e 2011).

Os ventos em $200 \mathrm{hPa}$ mostram um fluxo predominante de oeste para leste com o transporte de massa da Amazônia em direção do setor norte NEB, em particular nos dias -3 e -1 , com um auxílio de ventos de nordeste vindos do Atlântico Tropical, induzindo também um aporte do Oceano Atlântico (Figs. 2, 2c). Essa configuração de ventos de oeste em altos níveis antes de dias chuvosos na América do Sul, também se associa a propagação de Ondas de Kelvin e Rosby do Oceano Pacífico Tropical e latitudes médias para leste (Liebmann et al., 2011).

O campo de anomalias de geopotencial corrobora essa propagação de ondas de Kelvin e principalmente Rosby em latitudes médias que alternam centros de altas e baixas pressões que se deslocam desde áreas do Pacífico Subtropical Leste até a América do Sul e proximidades do Sul do Nordeste, típicos do padrão Pacífic South American (Souza e Ambrizzi, 2006).

A Fig. 3 mostra as anomalias dos ventos e temperatura em $850 \mathrm{hPa}$ para dias $-3,-2,-1,0 \mathrm{e}+1$ dos dias considerados chuvosos. Pode-se observar pelo campo de anomalias de ventos, que há uma intensificação dos alísios de nordeste nos dias -3 e -1 em relação a costa norte do Nordeste (Figs. 3a, e 3c), e que durante os dias -2 e) e +1 esses ventos enfraquecem embora ainda permaneçam soprando do Atlântico na direção da costa do NEB. Essa característica é muito típica dos meses mais chuvosos do setor semiárido do NEB, março a maio, quando os ventos alísios de nordeste mais intensos empurraram a ZCIT para próximo sobre a Costa norte do NEB, causando mais chuvas na região (Moura e Shukla, 1982; Nobre \& Shukla, 1996).
A essa intensificação dos ventos alísios antes dos dias chuvas intensas no NEB semiárido que transportando unidade do Oceano Atlântico Tropical, há uma incursão de ar frio (anomalias negativas de temperatura do ar) que se intensificam nos dias $-2 \mathrm{e}-1$, chegando a valores de inferiores a $-5^{\circ} \mathrm{C}$ e aumentam nos dias $0 \mathrm{e}+1$.

Kousky (1979) mostrou que a incursão de ar frio associado a penetração de sistemas frontais (Frentes Frias) para a região Nordeste, favorecem o abaixamento da pressão atmosférica nessa região, e essa massa de ar frio age também como um fator de ascensão dinâmica do ar quente e úmido junto a super superfície dessa região, favorecendo a convecção e formação de nuvens e precipitação.

Figuras não mostradas para anomalias de divergência de umidade e do vento em $850 \mathrm{hPa}$ mostraram valores negativos, indicando convergência de massa, para sobre o $\mathrm{NEB}$, principalmente entre os dias $-2 \mathrm{e}-1$. Nos dias $0 \mathrm{e}+1$ predominaram anomalias positivas dessas variáveis, indicativos de uma atmosfera mais estável, após os dias considerados chuvosos. Também advecção de temperatura em $859 \mathrm{hPa}$ (figuras não mostradas) evidenciaram uma incursão de advecção quente em áreas sobre o NEB e vizinhanças entre os dias - 3 e - 1 , antes do dia considerado chuvoso.

Nesses resultados foram mostrados apenas as figuras para o dias com precipitação, as configurações das Figs. 2 e 3, em geral, para os dias considerados secos, apresentaram características contrárias nas anomalias dessas variáveis.

\section{Conclusões e Recomendações}

Os resultados apresentados nesse estudo permitiram as seguintes conclusões.

- Quando ocorrem chuvas consideradas intensas no setor norte do NEB, principalmente sua região mais semiárida e de forma mais homogêneas, há uma configuração de ventos em altos níveis $(200 \mathrm{hPa}$ ) soprando de oeste com características associadas a propagação de ondas de Kelvin;

- Esse campos de ventos em $200 \mathrm{hPa}$, estiveram associadas a anomalias de ROL negativas mais intensas nessa faixa tropical do globo e sobre o NEB, mais evidentes nos dias -3 e - 1 . Nos dias chamados de 0 (de observação da precipitação acumulada das últimas 24 horas), e 1 após os eventos de precipitação essas características foram menos evidentes e anomalias de ROL passando a fracamente negativas;

- Anomalias de geopotencial em 200 hpa mostraram trens de ondas, similares a propagação de ondas de Rosby, entre os dias $-3 \mathrm{e}+1$, de origens subtropicais, se deslocando do Pacífico Leste em direção a América do Sul e NEB, indicando respostas a convecção ativa (não ativa) associada com centros de alta (baixa) pressão indicando divergência (convergência) de massa;

- No Atlântico Tropical, os resultados evidenciaram, pelas anomalias do vento em 850 hpa, implicitamente, a dinâmica de acoplamento oceano-atmosfera, associada ao 
dipolo negativo de TSM no Atlântico Tropical, que mostrou alísios de nordeste mais intensos, característica típica de quanto há um gradiente térmico apontando para o sul do equador, o que favorece a um posicionamento da ZCIT em latitudes mais próximas ou sobre a costa norte/nordeste do NEB;

- Outro resultado, bem evidente nos dias considerados chuvosos, entre fevereiro a maio, foi a incursão de uma
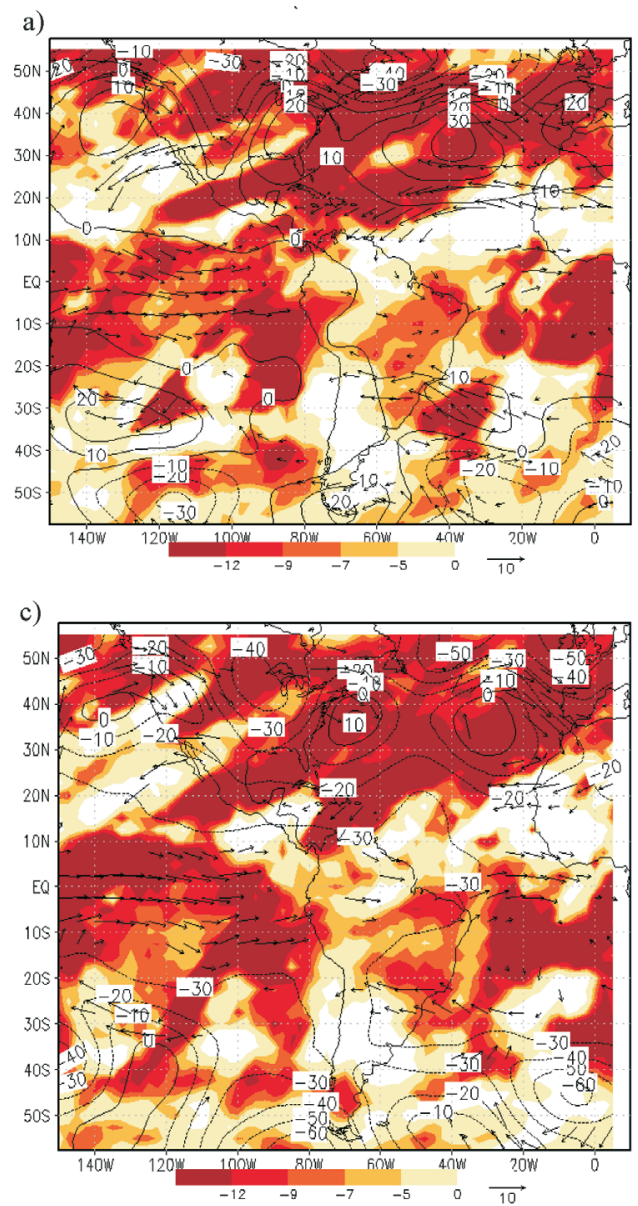

massa de ar frio, com valores de anomalias de temperatura do ar negativas, intensificando, entre o dia -3 e menos -1 , chegando a valores de menos $-5{ }^{\circ} \mathrm{C}$ sobre o NEB e vizinhanças. O que indicou que Frentes Frias, seu efeito de ascensão dinâmica, para o ar quente e úmido sobre a região, é um fator físico essencial para instabilizar a atmosfera e produzir precipitação mais intensa e homogênea sobre o NEB.
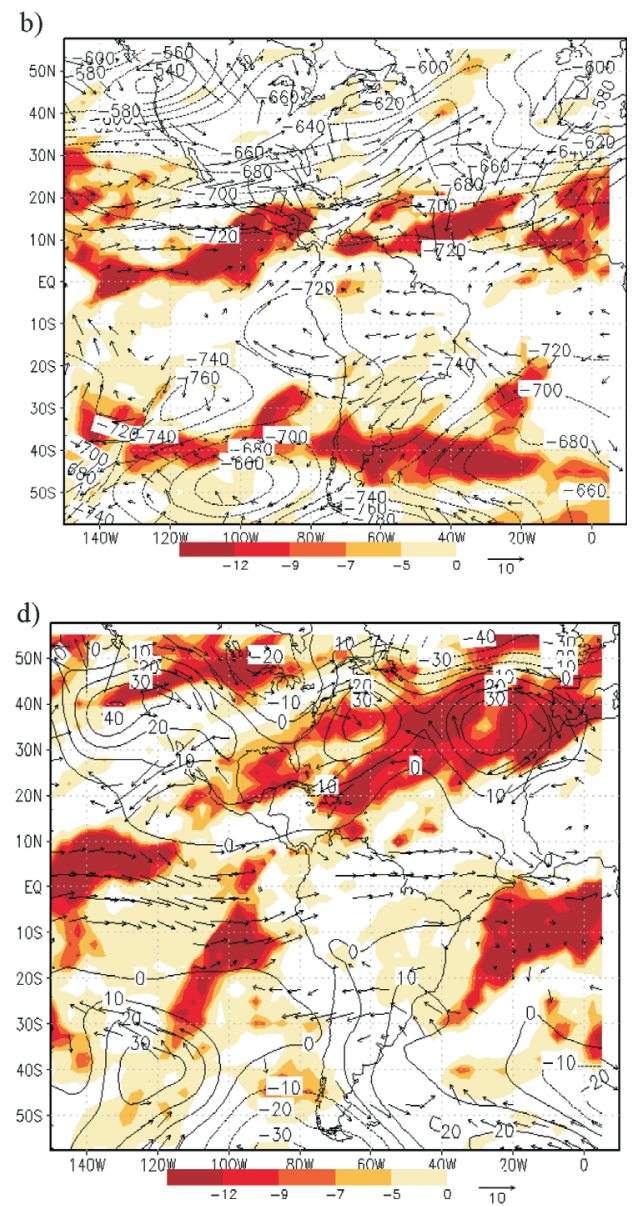

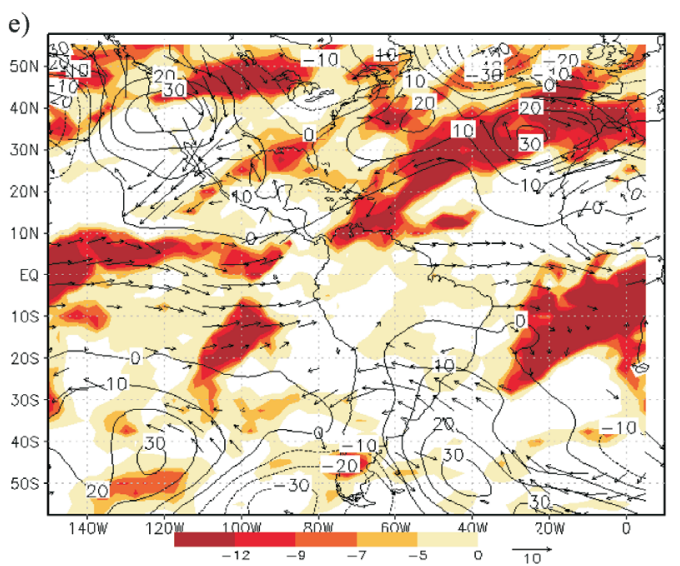

Figura 2 - Anomalias de ROL (W/m²), em cores, vento em $200 \mathrm{hPa}(\mathrm{m} / \mathrm{s})$ e geopotencial (m). a) dia -3, b) dia -2 , c) dia 0 , d) dia +1 , e) dia +2 , de dias considerados chuvosos no setor norte do NEB (Fig. 1), entre fevereiro a maio. Para as anomalias de ROL só anomalias negativas são plotadas. Foram plotadas apenas áreas com anomalias significativas a 95\% segundo teste $\mathrm{t}$ Student. 
a)

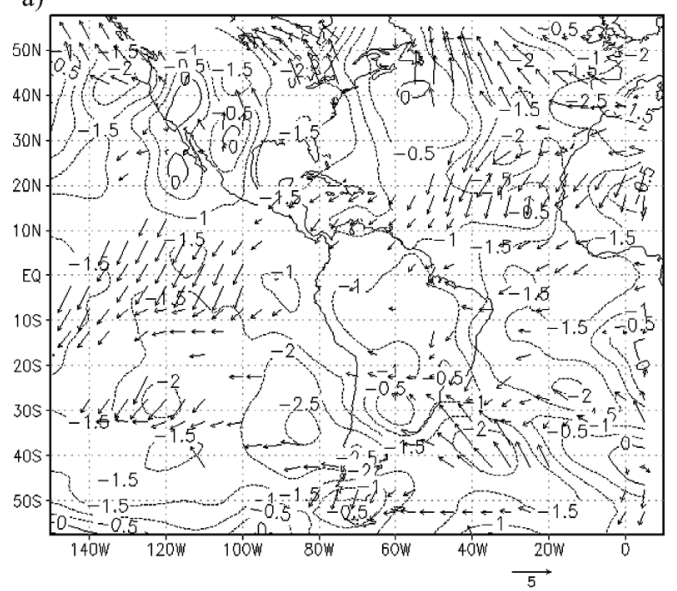

c)

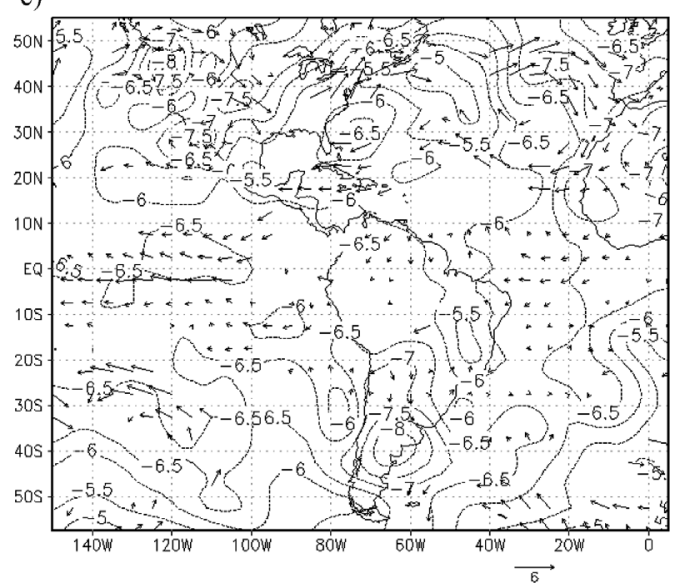

b)

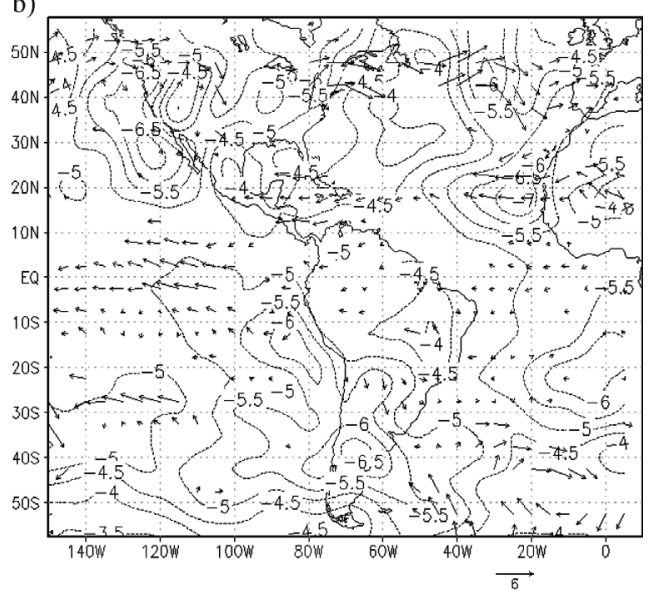

d)

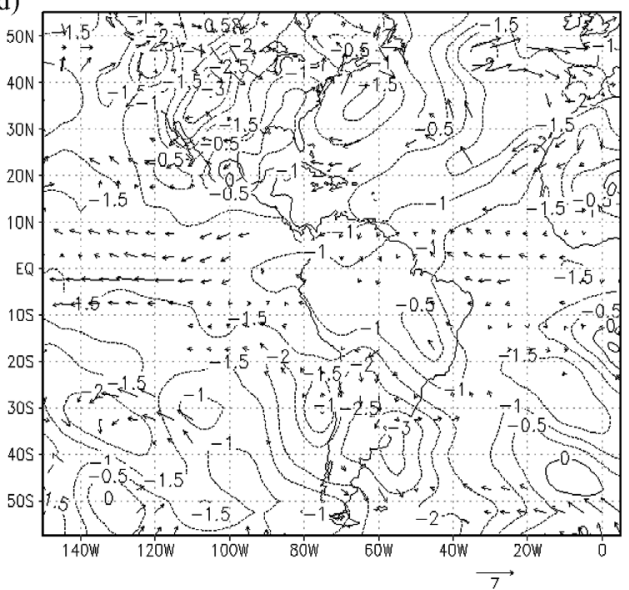

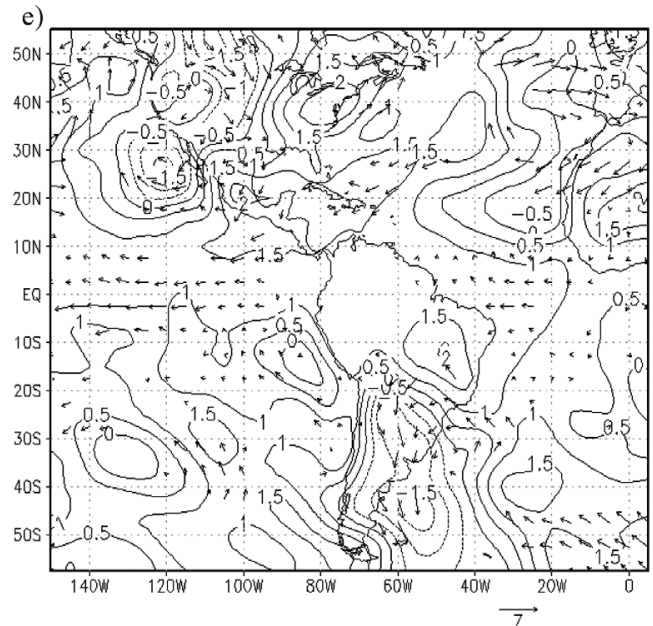

Figura 3 - Anomalias de vento em $850 \mathrm{hPa}(\mathrm{m} / \mathrm{s})$ e temperatura do ar média 1000-850 hPa $\left({ }^{\circ} \mathrm{C}\right)$. a) dia -3 , b) dia -2 , c) dia 0 , d) dia +1 , e) dia +2 , de dias considerados chuvosos no setor norte do NEB (Fig. 1), entre fevereiro a maio.

Para estudos futuros recomenda-se testar se os resultados de modelos que prognosticam a previsão de tempo sobre o NEB, ou rodadas climáticas de modelagem conseguem identificar esses padrões atmosféricos antes de eventos de precipitação mais intensa e homogênea sobre o NEB.

\section{Agradecimentos}

Os resultados apresentados nesse estudo são parte dos desenvolvidos sob fomento do Projeto - "Eventos Diários de Chuvas no Nordeste da América do Sul e suas Características Atmosféricas, Processo: 471814/2013-0, Chamada: Universal 14/2013 - Faixa A - CNPq, sob a coordenação de José Maria Brabo Alves. 


\section{Referências}

ALVES, J.M.B.; SERVAIN, J. \& CAMPOS, J.N.B. Relationship Between Ocean Climate Variability and Rain Fed Agriculture in Northeast Brazil. Clim Res., v. 38, p. 225-236, 2009.

ALVES, JOSÉ M. BRABO et al. Mecanismos atmosféricos associados à ocorrência de precipitação intensa sobre o Nordeste do Brasil durante Janeiro/2004. Revista Brasileira de Meteorologia, v. 21, n. 01, p. 1-21, 2006

CARVALHO, L.M.V.; JONES, C. \& LIEBMANN, B. The South Atlantic Convergence Zone. Intensity, form, persistence, and relationshisp with intra-seasonal to interannual activity and extreme rainfall. J Clim, Boston, v. 17, p. 88-108. 2004.

GARREAUD, R. D. 2000 Cold air incursions over subtropical South America: Mean structure and dynamics. Mon. Wea. Rev., 128, 2544-2559.

GARREAUD, R. D AND WALLACE, M. J. 1998 Summertime incursions of mid-latitude air into subtropical and tropical South America. Mon. Wea. Rev., 126, 2713-2733.

HASTENRATH，S. \& GREISCHAR，L. Circulation mechanisms related to northeast Brazil rainfall anomalies. J. Geophy. Res., v. 98, p. 5093-5102. $1993 b$.

KALNAY, E., and Coauthors, 1996 The NCEP/NCAR 40-Year Reanalysis Project. Bull. Amer. Meteor. Soc., 77, 437-471.

KOUSKY, V. E. \& FERREIRA, N. J. 1981 Interdiurnal surface pressure variations in Brazil: Their spatial distributions, origins, and effects. Mon. Wea. Rev., 109, 1999-2008.

KOUSKY, V. E. Frontal influences on Northeast Brazil. Monthly Weather Review, 107(9), p.1140-1153, 1979.

LIEBMANN, B.; KILADIZ, G.N.; ALLURED, D.; VERA, C.; JONES, C.; CARVALHO, L.M. V; BLADE, I.B. \& GONZALES, P.L.M. 2011. Mechanisms Associated with Large Daily Rainfall Events in Northeast Brazil. Journal of Climate, DOI: 10.1175/2010JCLI3457.1

LIEBMANN, B.; KILADIZ, G.N.; CARVALHO, L.M.V.; JONES, C.; VERA, C.S.; BLADE, I. B. \& ALLURED, D. Origin of convectively coupled Kelvin waves over South America. J. Climate, v. 22, p. 300-315. 2009.

MORAN, V.; ROBERTSON, A.W.; WARD, M.N. \& CAMBERLIN, P. Spatial coherence of tropical rainfall at the regional scale. J. Climate, v. 20, p. 5244-5263. 2007.

Moura, A.D. \& Shukla, J. On the dynamics of droughts in northeast Brazil: Observations, theory and numerical experiments with a general circulation model. J. Atmos. Scie., v. 38, n. 12, p. 2653-2675. 1981.

NOBRE, P. \& SHUKLA, J. Variations of sea surface temperatures, wind stress, and rainfall over the tropical over the tropical Atlantic and South America. J. Climate., v. 9, n. 10, p. 2464-2479. 1996.

RAMOS, R. P. L. 1975 Precipitation characteristics in the Northeast Brazil dry region. J. Geophys. Res., 80, 1665-1678.

RAO, V. B.; FRANCHITO, S. H. \& BARBOSA, T. F. 2007 Impact of high potential vorticity intrusions into the tropical upper troposphere in South Atlantic on precipitation over Northeast Brazil. Geophys. Res. Lett., 34, L06704, doi:10.1029/2006GL027873.

RIECKENBACH, T. M.; FERREITA, R. N.; HALVERSON, J. B.; HERDIES, , D. L. \& SILVA, DIAS, M. A. F. 2002 Modulation of convection in the southwestern Amazon basin by extratropical stationary fronts. J. Geophys. Res., 107, 8040, doi:10.1029/2000JD000263.

ROPELEWSKI, C.F. \& HALPERT, M. Global and regional scale precipitation patterns associated with the El Niño/Southern Oscillation. Monthly Weather Review, v. 115, p. 1606-1626. 1997.

ROPELEWSKI, C.F. ; \& JONES, P.D. An Extension of the Tahiti-Darwin Southern Oscillation Index. Mon. Wea. Rev., v. 15, p. 2161-2165. 1987.

SOUZA, E.B. \& AMBRIZZI, T. Modulation of the intraseasonal rainfall over tropical Brazil by the Madden-Julian oscillation. International Journal of Climatology, v. 26, p. 1759-1776. 2006.

SOUZA, E.; KAYANO, M.T. \& AMBRIZZI, T. Intraseasonal and submonthly variability over the eastern Amazon and Northeast Brazil during the autumn rainy season. Theor. Appl. Climatol., v. 81, p. 177-191. 2005.

UVO, C.B.; REPELLI, C.A,; ZEBIAK, S.E. \& KUSHNIR, $Y$. The relationships between tropical Pacific and Atlantic SST and Northeast Brazil monthly precipitation. J. Climate, v. 11, p. 551-562. 1998.

VERA, C.S. \& VIGLIAROLO, P.K. A diagnostic study of cold-air outbreaks over South America. Mon. Wea. Rev., v. 128, p. 3-24. 2000.

This is an Open Access article distributed under the terms of the Creative Commons Attribution Non-Commercial License which permits unrestricted non-commercial use, distribution, and reproduction in any medium provided the original work is properly cited. 